\title{
A proposed new design for a large radio telescope
}

\author{
T.H. Legg \\ Herzberg Institute of Astrophysics, National Research Council of Canada, 100 Sussex Drive, Ottawa, K1A 0R6, Canada
}

Received March 11; accepted December 2, 1997

\begin{abstract}
A new type of radio telescope is proposed which may make very large telescopes more affordable. The telescope is considered to be one of several identical elements that form a synthesis array. It is composed of an almost flat primary reflector that is slightly adjustable in shape and made up of identical square flat panels supported by the ground. A very long focal length imposes the unusual condition that the receiver be carried by an airborne vehicle such as a powered, helium-filled balloon. The position of the balloon is measured and controlled as accurately as possible and residual errors in the balloon's position are dealt with in two ways. Errors of a few metres are corrected by moving the receiver feed point electronically. Larger errors are corrected by adjusting the primary reflector so as to move its focal point to follow the balloon. These features maintain telescope efficiency and correct pointing so long as the balloon lies anywhere within a large volume surrounding its ideal position. The problem of controlling the balloon position is thereby substantially eased. The telescope has the wide sky coverage needed for synthesis observations and an estimated optimum diameter in the range 100 to $300 \mathrm{~m}$. It will operate from decimetre to short centimetre wavelengths, or, with smaller panels, millimetre wavelengths.
\end{abstract}

Key words: telescopes - instrumentation: interferometers

\section{Introduction}

There is a clear scientific need for a radio telescope some 10 to 100 times larger in area than existing telescopes. Such an instrument is the subject of an International Union of Radio Science (URSI), commission J (radio astronomy) study (Braun 1993, 1996) and would have a major impact in nearly every area of radio astronomy. It would greatly increase the number of observable radio sources of types

Send offprint requests to: T.H. Legg already investigated as well as opening up entirely new fields such as, for example, the study of nearby stars similar to the sun or of galaxy formation at very large redshift. It could also greatly increase the range and capability of radar astronomy.

A single very large collecting area would, by itself, lack angular resolution and be confusion limited for many observations and therefore inappropriate. Instead, a number of telescope elements will have to be joined interferometrically to synthesize a larger aperture. The need for aperture synthesis implies that long baseline tracks are required and that the individual telescope elements must be steerable over a large part of the sky. A telescope array with this capability can then also be combined with existing telescopes used for Very Long Baseline Interferometry (VLBI), including telescopes in space. Amongst the benefits would be a great improvement in VLBI polarization measurements which would bear on the understanding of the physical structure of jets in active galactic nuclei and other objects (Gabuzda et al. 1992; Kemball et al. 1996). It might also allow observation of the transverse velocity component of $\mathrm{H}_{2} \mathrm{O}$ masers orbiting in external galaxies. This observation, when compared statistically with spectroscopically determined radial velocities, could lead to unbiased distance estimates for external galaxies (Reid \& Moran 1988).

Reber (1940) built the first radio telescope to use a paraboloidal reflector almost 60 years ago. Fully steerable paraboloids have subsequently been the mainstay of radio astronomy for wavelengths ranging from metres to submillimetres. As a result of this widespread use, construction of steerable paraboloids has been well optimized to minimize cost and not very much additional saving can be expected.

Very little of the cost and weight of a steerable paraboloid is in the reflecting surface itself. The part that is expensive is the structure needed to support and move the reflector. It is clear that there are potentially very large savings in keeping the reflector close to the ground and supported by the ground and by avoiding the heavy 


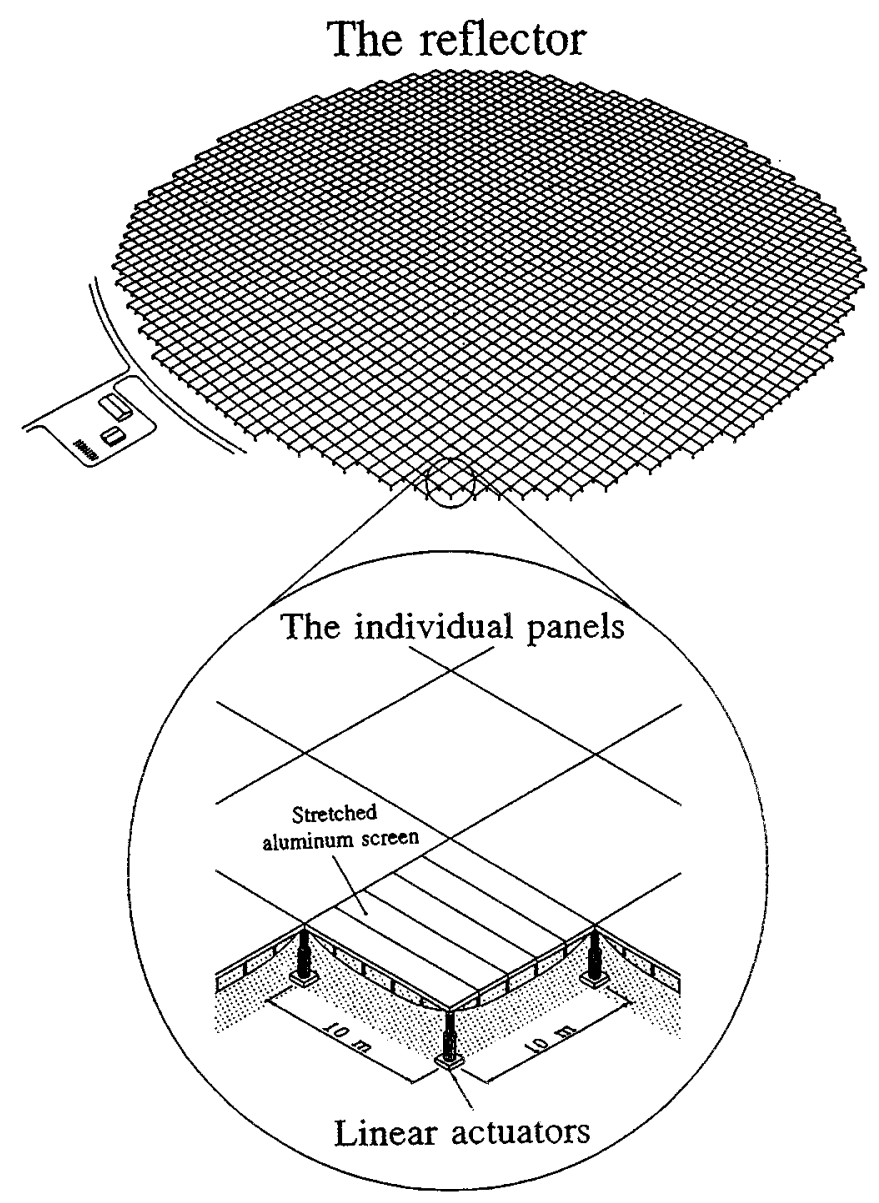

Fig. 1. A large, almost flat primary reflector is supported by the ground and slightly alterable in shape through the adjustment of linear actuators

rotating machinery of a steerable paraboloid. This was done with the Ohio State telescope (Kraus \& Nash 1961), the Ratan 600 telescope in Russia (Korolkov \& Pariiskii 1979), the Arecibo telescope (Gordon \& Lalonde 1961), and Nançay telescope (Blum et al. 1963). All of these telescopes have provided large collecting areas relatively inexpensively, though at the price of limited sky coverage and therefore limited usefulness in aperture synthesis.

A different type of reflecting telescope is discussed here which may provide an economical means of achieving both a very large collecting area and the good sky coverage needed for modern aperture synthesis. Some early ideas on the scheme have already been briefly outlined (Legg 1996).

\section{How it would work}

We will discuss here just one of several identical telescopes that together make up a synthesis array. This single telescope consists of a primary reflector composed of a large number of identical square panels as sketched in Fig. 1.

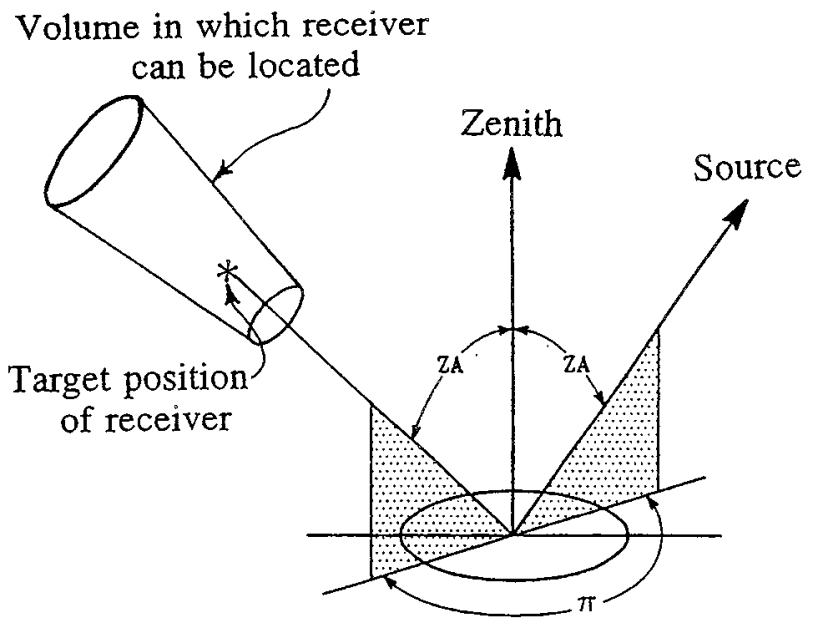

Fig. 2. The telescope receiver is carried by an airborne vehicle and driven towards the target position shown here. As a result of corrections to the receiver feed point and reflector adjustments which move the diffraction image, telescope efficiency and correct pointing are maintained with the receiver anywhere within a relatively large volume surrounding the target position

The panels are supported at their corners by linear actuators which in turn are supported by concrete footings set in the ground. Both the panels and the overall reflector are almost flat, with the reflector being slightly alterable in shape through adjustment of the linear actuators. With this arrangement the reflector is close to the ground and its panels can be large, identical, flat, and inexpensive. In particular, it is believed that the panels would be substantially less expensive per unit area than the doubly-curved panels needed in an Arecibo type of telescope. However, as a result, the focal length of the reflector needs to be very long. This imposes the unusual condition that the radio receiver be carried by some form of airborne vehicle.

Emission from a radio source is focussed by the reflector to form a diffraction image. Ideally, this image is at a target position as shown in Fig. 2. This position is at the zenith angle of the source and at an azimuth $180^{\circ}$ from the source azimuth. The reflector at all times forms part of a paraboloid and has no appreciable path-length errors or aberrations. To maintain these conditions the reflector is continuously adjusted with the linear actuators so that the particular paraboloidal section being used remains suited to the changing azimuth and zenith angle of the source. Wide sky coverage is thereby achieved, though the collecting area is reduced by foreshortening at large zenith angles (by a factor of two, for example, at a zenith angle of $60^{\circ}$ ). This reduction, and a corresponding change in the primary beam shape with zenith angle, would also be encountered with the phased-array type of telescope being studied for the one-square kilometer telescope (Braun 1993). With both the presently proposed telescope and the phased array, the primary collecting element is fixed 
to the ground. This ensures that accurate account can be taken of the foreshortening effect in processing a synthesized image.

The position of the airborne vehicle is measured and controlled from the ground to keep the receiver feed in alignment with the diffraction image as closely as possible. Any displacement of the vehicle from this alignment is dealt with in two ways. Large displacements $( \pm 15 \mathrm{~m}$, for example), are compensated by adjusting the primary surface actuators so as to move the focussed diffraction image of the source to follow the vehicle. This greatly eases the problem of controlling the vehicle position by allowing it to be located anywhere within a large volume surrounding the target position as shown in Fig. 2. This may be a feature which makes the telescope scheme practicable. Moving the diffraction image to follow the vehicle does not, however, remove all of the effects of vehicle motion. For a $200 \mathrm{~m}$ telescope considered below as an example (Table 1), a $15 \mathrm{~m}$ displacement of the vehicle can change the telescope collecting area by up to $1 \%$ as a result of changed foreshortening. Illumination efficiency can also change by about $1 \%$. These effects would need to be corrected in the image processing.

At short wavelengths, where accurate pointing is specially important, smaller vehicle displacements of a few metres are compensated by an equal and opposite displacement of the area over which source energy is collected, thereby keeping the pointing constant. This is done essentially instantaneously using a phased receiving array and allows the surface actuator adjustments to be made more slowly and less often. (At $\mathrm{mm}$ wavelengths the phased array is replaced with a small mechanically swivelled mirror). At longer wavelengths, where the size of the diffraction image is large, small vehicle displacements are less important and are tolerated. However, large displacements are still corrected by moving the focal point using the surface actuators. The longer wavelength observations are made by reducing the altitude of the vehicle and re-focusing the primary reflector so as to operate with a smaller $f / D$ ratio.

For $\mathrm{mm}$ wavelengths, a large $f / D$ ratio gives the opportunity of constructing very accurate flat panels inexpensively from thin stretched sheets of metal. The stable support of each panel by the ground avoids the need for a protective radome.

\section{Detailed features and telescope examples}

The concept of the proposed telescope is believed to be sound in featuring: (i) a very long focal length to allow economical and stable support of the reflecting surface by the ground, (ii) the use of inexpensive flat panels, and (iii) small surface adjustments to avoid path-length errors over a wide area of sky. However, these advantages come only with the risk of adopting an unproven and very unconventional scheme of supporting the receiver with an airborne
Table 1. An example, a 200 m centimetre-wavelength telescope

\begin{tabular}{ll}
\hline Azimuth range, deg. & 360 \\
Elevation range, deg. & 90 to 30 (for half power) \\
Reflector diameter, m & 200 \\
Wavelength range, cm & 1.3 to 35 \\
Beamwidth, arcmin & 0.25 to 6.7 \\
Focal length, $Z A=0, \mathrm{~m}$ & 1500 to 3000 \\
Panel dimension, m & 10 \\
Number of panels & 312 \\
Number of actuators & 352 \\
Actuator travel, m & 2.1 \\
Actuator accuracy, mm & 1.0 \\
Secondary diameter, m & $8(\lambda \geq 10 \mathrm{~cm})$ \\
& or $4(\lambda \leq 10 \mathrm{~cm})$ \\
\hline
\end{tabular}

vehicle. Examples of $\mathrm{cm}$ and $\mathrm{mm}$ wavelength telescope elements are described in this section with characteristics listed in Tables 1 and 2. The suggested construction features are not necessarily optimum but are discussed in enough detail to illustrate how the general telescope scheme, including its airborne receiver, might be made workable.

It is believed that telescopes of this type would be least expensive per unit of collecting area when their diameters range from about $100 \mathrm{~m}$ to $300 \mathrm{~m}$. The $200 \mathrm{~m}$ example of Table 1 may be close to an optimum. An upper limit on diameter is estimated to be about $500 \mathrm{~m}$. This is set by an increase in size and cost of the surface actuators with the reflector diameter and by a limit in how high the receiver can be carried. A lower economic limit on size is set by the fixed expense of the airborne vehicle.

The optimum wavelength, again from the point of view of cost, appears to be in the cm range. Longer wavelengths require shorter focal lengths which become progressively more expensive. Millimetre wavelength operation requires smaller, more accurate panels, but seems to be entirely practical provided that the rapid electronic correction of pointing (which is also needed at $\mathrm{cm}$ wavelengths) is effective. Several options for individual mm-wave telescopes are listed in Table 2. For the purpose of comparison, each of these telescopes requires about 2000 actuators. An alternative to a separate mm-wave telescope would be to use smaller mm-wave panels over part of the area of a longer wavelength reflector.

\subsection{Some constraints and relationships}

It is useful to define several quantities and their relationship before discussing the telescope features in detail. Symbols that will be used are:

$\lambda$, the observing wavelength

$D$, the diameter of the primary reflector

$L$, the side length of a square primary reflector panel

$S$, the diameter of the secondary reflector 
Table 2. Examples of millimetre-wave telescopes with about 2000 actuators

\begin{tabular}{lllll}
\hline Azimuth range, deg. & \multicolumn{4}{l}{360} \\
Elevation range, deg. & 90 to & \multicolumn{3}{l}{ (for half power) } \\
Reflector diameter, m & 125 & 110 & 80 & 65 \\
Shortest $\lambda, \mathrm{mm}$ & 3.5 & 2.6 & 1.3 & 0.87 \\
Beamwidth, arcsec & 6.9 & 5.8 & 4.9 & 4.9 \\
Focal length, $Z A=0, \mathrm{~m}$ & 3000 & 3000 & 3000 & 3000 \\
Panel dimension, m & 2.6 & 2.3 & 1.6 & 1.3 \\
Number of panels & 1820 & 1800 & 1960 & 1960 \\
Number of actuators & 1890 & 1870 & 2040 & 2040 \\
Actuator travel, cm & 22.6 & 17.5 & 9.3 & 6.1 \\
Actuator accuracy, mm & 0.26 & 0.20 & 0.10 & 0.08 \\
Secondary diameter, m & 4 & 4 & 4 & 4 \\
\hline
\end{tabular}

$R$, the primary to secondary reflector distance

$Z A$, the zenith angle of the radio source

$h=R \cos (Z A)$, the altitude of the receiver

$A$, the motion or travel of the actuators used to adjust the primary surface

$W$, the diameter of the region through which the actuators move the focus.

The actuator travel, $A$, is an important cost consideration and constrains the maximum telescope size and lowest operating frequency. The actuators do three things: adjust the shape of the primary surface to suit the position of the radio source, change the focal length of the primary reflector to suit the observing wavelength, and move the focus to follow unprogrammed motion of the airborne vehicle within a volume of diameter $W$. It is found that at a given observing wavelength, actuator travel is minimized by keeping the airborne vehicle at a constant altitude. Under this condition, the maximum actuator travel required is:

$$
\begin{aligned}
A= & \left(D^{2} / 16\right)\left(h_{\min }^{-1}-h_{\max }^{-1} \cos ^{2} Z A_{\max }\right) \\
& +(W D / 8)\left(h_{\min }^{-1}+h_{\max }^{-1} \cos Z A_{\max }\right) .
\end{aligned}
$$

The first term represents the adjustment needed to focus at the minimum operating altitude $\left(h_{\min }\right)$ and zero zenith angle compared to the maximum altitude $\left(h_{\max }\right)$ and maximum zenith angle $\left(Z A_{\max }\right)$; the second term the adjustment to follow the receiver over a lateral movement of $W$ metres. A good approximation to Eq. (1) is found to be:

$A \approx\left(h_{\min }^{-1} / 8\right)\left(W D+D^{2} / 2\right)$

which shows that actuator travel is roughly inversely proportional to the minimum altitude of the receiver. The number of actuators required is approximately:

$N_{\mathrm{a}} \approx(\pi / 4)(1+D / L)^{2}$

and the number of primary reflector panels is approximately:

$N_{\mathrm{p}} \approx(\pi / 4)(D / L)^{2}$.
There is a clear advantage in making the panel size as large as possible to minimize the number of panels and actuators. Flat, or nearly flat panels are required and a limit on their size is imposed by differences in path-length to the receiver at the shortest observing wavelength (Sect. 3.6). The relationship between the largest panel size and shortest observing wavelength is taken to be:

$L \leq(\lambda R)^{1 / 2}$.

The diameter between the first zeroes in the primary reflector diffraction image of a small radio source (see, for example, Silver 1949) is $4.88 \lambda R / D$ for $Z A=0$. Telescope efficiency begins to fall with increasing wavelength when this diameter becomes larger than the secondary reflector. At this point, foreshortening can also have an effect by elongating the image in the source direction. The fall-off in efficiency is gradual, however, and we take as a longest wavelength limit the wavelength at which efficiency has dropped by about a factor of two at a zenith angle of $60^{\circ}$. This gives:

$\lambda \leq 0.32 D S / h_{\text {min }}$.

This wavelength limit is increased by making the receiver altitude as small as possible. The degree to which this can be done, however, is limited by the actuator travel described by expressions (1) and (2). Combining expressions (2) and (6) to eliminate $h_{\min }$ and taking the maximum zenith angle to be $60^{\circ}$ gives:

$\lambda \leq 5.1 A S /(D+2 W)$.

These relationships are used in drawing Fig. 3 where three distinct operating ranges of the telescope are indicated. These go from short centimetre (or, with smaller panels, millimetre) wavelengths to decimeter wavelengths. In range (a) the telescope is operated with the receiver at maximum altitude in order that the primary reflector panels can be as large as path-length differences allow (expression 5). The diffraction image is small compared to the secondary reflector. In range (b) the receiver distance is reduced in proportion to $\lambda^{-1}$ to keep the image smaller than the secondary, thereby continuing to allow rapid pointing correction with a phased receiving array. In region (c), the telescope is operated at the lowest altitude allowed by the actuator travel. Increasingly longer wavelengths are observed until the secondary reflector intercepts too small a fraction of the source diffraction image. Small pointing errors are tolerated because of the larger diffraction image while larger errors are still corrected by moving the surface actuators.

\subsection{The airborne vehicle}

The airborne vehicle is clearly the key component of the telescope. Remotely controlled helicopters are possible choice because of their potentially good positional and attitude stability. An example is the Canadair CL-227, a 


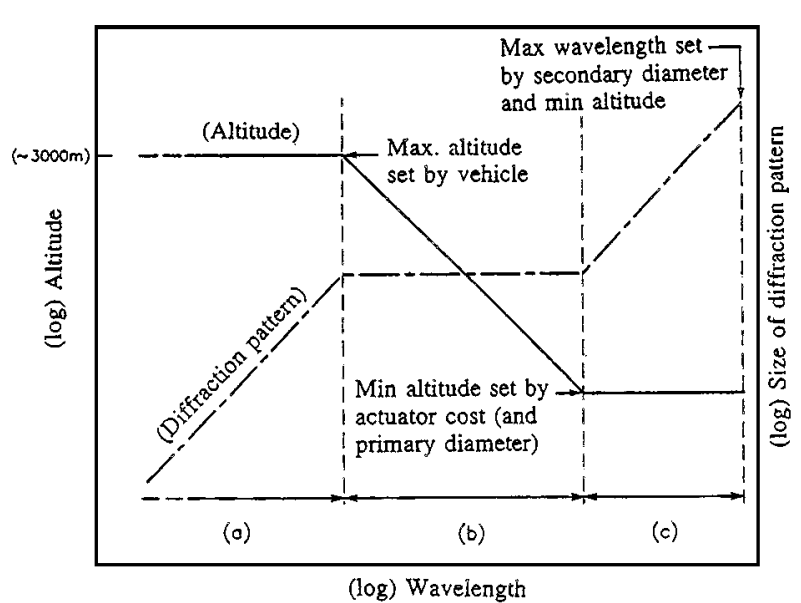

Fig. 3. Three operating regions of the telescope

small military surveillance helicopter now no longer being built. Estimates of the stability of this vehicle were made from images of the ground obtained with an on-board television camera and were limited by the camera resolution at higher altitudes (Clark 1988). The estimated instability in position was 2 to 3 meters at low altitudes possibly increasing to a few tens of meters at high altitudes (maximum altitude was $3000 \mathrm{~m}$ ). Attitude was controlled by on-board sensors which gave a stability of better than 0.1 degrees per hour. With the scheme discussed here of moving the diffraction image to follow vehicle motion over a few tens of meters, this helicopter stability would evidently be sufficient to ensure a functioning radio telescope.

It is believed, however, that a powered, helium-filled balloon could also be made sufficiently stable while offering lower initial and operating costs and better reliability. Several such machines have recently been built with spherical envelopes of diameter $5.8 \mathrm{~m}$ and $13.1 \mathrm{~m}$ (Colting 1994). These balloons are operated remotely by radio link, can be handled on the ground by a crew of two and do not require a hanger. It is estimated that a $10 \mathrm{~m}$ diameter balloon would have sufficient lifting capacity for the receivers and feeds of the proposed telescope. There is a large saving in the cost if, as for the present application, the balloon does not have to be certified to carry people.

The existing balloons are powered by two piston engines placed on opposite sides of the balloon and below the centre. Altitude is controlled by tilting the propellers in a vertical plane while differential thrust from the propellers turns the balloon about a vertical axis. Horizontal vanes are placed behind the propellers and can be adjusted in angle, differentially on the two sides if need be, to deflect the propeller slipstream up or down.

The existing balloons have a control scheme that has been proven in practice but it does not provide the full, so-called six-axis control of position and attitude which would be needed for the proposed telescope. Six-axis control can evidently be achieved, however, by adding a set of vertical vanes, together with an extra set of horizontal

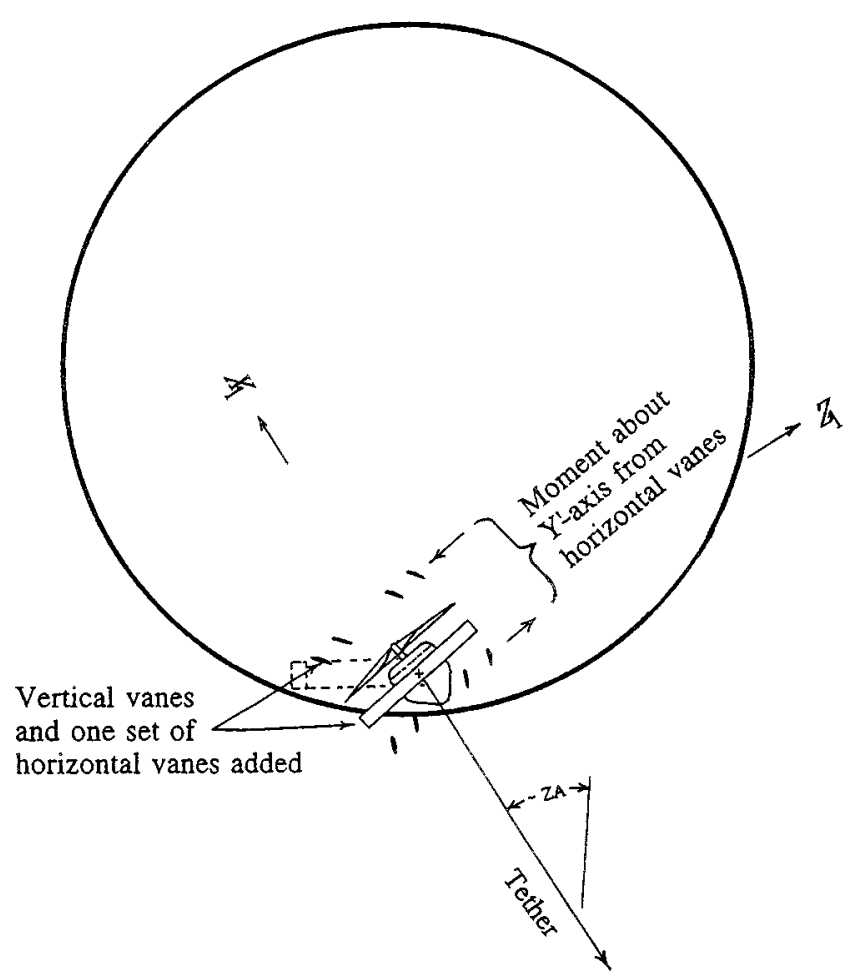

Fig. 4. Engines, propellers and slipstream-deflecting vanes are situated on both sides of the balloon and control its position and attitude

vanes, this latter set in front of the propellers as sketched in Fig. 4.

It may also be advantageous to add a tether to the balloon. The tether would provide a steady force that would be counteracted by propeller thrust. The benefit of this is that the control vanes could then rapidly deflect an already existing propeller slipstream. The ground end of the tether would be anchored on a winch slightly to the north or south of the primary reflector to avoid entanglement with the reflector panels. Displacements of the vehicle in the direction of the tether would be adjusted by propeller thrust and by controlling the unreeling of the tether from the ground. Relatively large errors in position are tolerable in this direction because the large $f / D$ ratio of the telescope gives a large depth-of-field (about $\lambda(f / D)^{2}$ ). A suitable tether would be similar to one recently used with instrument carrying kites (Beard 1994). This tether, made from Kevlar, is $6 \mathrm{~km}$ in length, withstands a load of $430 \mathrm{~kg}$ and weighs $18 \mathrm{~kg}$.

As in the balloons already built, the propellers and vanes will be tiltable in the vertical plane. This will allow the propeller thrust to be aligned approximately antiparallel to the force from the tether. Tilting the propellers slightly out of this alignment can then provide a steady thrust component that will help to control the balloon altitude. With an $x_{1}$ axis taken to be in the tether direction (Fig. 4), control forces are provided in the $x_{1}$ direction by propeller thrust, in the $y_{1}$ direction by the vertical vanes 
(or, more slowly, the engine tilt), and in the $z_{1}$ direction by the horizontal vanes. Turning moments about these axes are provided by a difference in force from the horizontal vanes on opposite side of the balloon $\left(x_{1}\right)$, by a differences in force from horizontal vanes in front of and behind the propellers $\left(y_{1}\right)$, and by a difference in thrust from the two propellers $\left(z_{1}\right)$. The coupling of some of these control forces and moments will have to be compensated in the control software. For example, to keep a turning moment constant, the angle of attack of a vane will have to be adjusted when propeller thrust is changed.

The forces and power involved in the control of the balloon can be readily estimated. The mass of a balloon in hydrostatic equilibrium is equal to the mass of displaced air. For a standard air density of $0.909 \mathrm{~kg} \mathrm{~m}^{-3}$ at $3000 \mathrm{~m}$ altitude and a spherical $10 \mathrm{~m}$ diameter balloon, the equilibrium mass is $476 \mathrm{~kg}$. For air of this density moving past a $10 \mathrm{~m}$ diameter sphere at $30 \mathrm{~km}$ per hour, the aerodynamic drag coefficient is about 0.2 . These numbers imply that a sudden gust of $30 \mathrm{~km}$ per hour will exert a force of $4.910^{7}$ dynes on the balloon which, if not counteracted by a control force, will result in an acceleration of $1 \mathrm{~m}$ per $\mathrm{sec}^{2}$ and a displacement of $0.5 \mathrm{~m}$ in the first second after the onset of the gust. The power required to produce $4.910^{7}$ dynes of thrust at $75 \%$ propeller efficiency and an airspeed of $30 \mathrm{~km}$ per hour is $5.5 \mathrm{kw}$. Similarly, counteracting a wind of $50 \mathrm{~km}$ per hour will require $15 \mathrm{kw}$. However, we note that about three times as much power is needed to produce a given force with the control vanes if these are able to divert the slipstream through an angle of only, say, 20 degrees. We also note that the weight of small aircraft piston engines (for example, those used in ultralight aircraft) is about $0.6 \mathrm{~kg}$ per $\mathrm{kw}$.

The numbers mentioned above, the existence of balloons not drastically different from ones that would be needed, and the large error in balloon position that can be tolerated by adjusting the telescope focus position, are encouraging. However, the first step needed in establishing the feasibility of the proposed telescope would be to actually demonstrate a balloon with a suitable control scheme.

\subsection{Telescope feed antennas}

The size of the telescope diffraction pattern from a small source is listed in Table 3 for several wavelengths and for zenith angles of $0^{\circ}$ and $60^{\circ}$. The numbers are the diameters between the tenth power points in the diffraction pattern of a small, unresolved source. They give some idea of the effective size of the telescope feed antenna needed at the different wavelengths and zenith angles and show the change in shape needed, for an ideal feed, with changing zenith angle.

This change could, in principle at least, be provided by a large phased receiving array whose active area is controlled to match the area of the diffraction image. The
Table 3. Size of the diffraction image at the feed antenna

\begin{tabular}{llll}
\hline$\lambda$ & $\begin{array}{l}\text { Balloon } \\
\mathrm{cm}\end{array}$ & $\begin{array}{l}1 / 10 \text { power diameters, } \mathrm{m} \\
\left(\mathrm{Z} A=0^{\circ}\right.\end{array}$ & $Z A=60^{\circ}$ \\
\hline 1.3 & 3000 & $0.46 \times 0.46$ & $0.91 \times 1.8$ \\
3.2 & 3000 & $1.1 \times 1.1$ & $2.2 \times 4.5$ \\
10 & 1500 & $1.7 \times 1.7$ & $3.5 \times 7.0$ \\
21 & 1500 & $3.7 \times 3.7$ & $7.4 \times 15$ \\
35 & 1500 & $6.8 \times 6.8$ & $12.3 \times 24$ \\
\hline
\end{tabular}

angle of reception of the array and position of its active area could also be adjusted electronically to suit the source zenith angle and to correct for some of the unprogrammed motion of the supporting balloon. However, each array element requires its own amplifier, attenuator and phase shifter and even at a wavelength as long as $10 \mathrm{~cm}$, about 10000 elements would be needed for two polarizations. Such an array may be possible in the future but would require substantial development.

A feed antenna that would provide most of the same adaptability with existing technology would make use of a much smaller phased array illuminating a large secondary reflector as sketched in Fig. 5. The feature that is not immediately provided is the change in the angle of reception to suit the source zenith angle. Ideally, this could be achieved over a $60^{\circ}$ zenith angle range by tilting the whole balloon $\pm 30^{\circ}$ from the vertical. However, if this is not practical, the array would need to be either moved along a track or extended as sketched in Fig. 5 .

A phased array feed has the additional advantage that the illumination could be made relatively constant over the primary reflector, dropping off rapidly at the edge. This will reduce illumination spillover to give high aperture efficiency. The primary beam sidelobe level will be higher but this is entirely acceptable for an element of a synthesis array. Small spillover also limits the $300 \mathrm{~K}$ ground radiation that reaches the receiver which could otherwise be worse than with a conventional steerable paraboloid.

For the longer wavelengths, the secondary reflector of the proposed feed is $8 \mathrm{~m}$ in diameter and made of a light fabric. The reflector shape is determined by the shape of the individual sectoral gores which compose it, and by a small overpressure on one side to keep the fabric under tension. A reflector with a more accurate, rigid surface will be needed for shorter wavelengths. It is believed that a suitable surface might be made primarily from plastic foam with a honey-comb backing of thin foam sections. Because of the smaller diffraction image at short wavelengths this secondary can be smaller. It would be an aim to make this reflector $4 \mathrm{~m}$ in diameter and to have it form part of the larger fabric reflector. This rigid reflector will be supported by the (offset) balloon framework which joins the two engine mounts. 


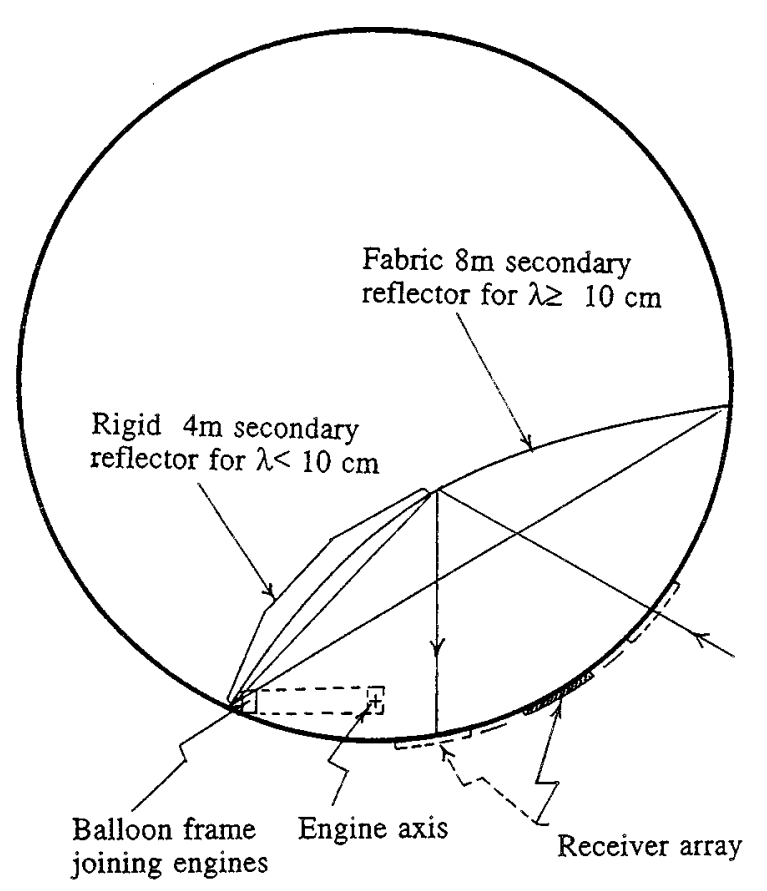

Fig. 5. The telescope feed antenna consists of a phased receiving array and a secondary reflector. The fabric part of the secondary is used at long wavelengths. At short wavelengths the phased array illuminates a relatively small area on the rigid part of the secondary. This area can be moved to compensate for balloon motion and maintain accurate pointing

At the shorter wavelengths, where the diffraction image of an unresolved source is smaller than the secondary, the small phased receiving array will illuminate an area of the secondary the size and shape of the image. If the position of the image moves on the secondary, as a result of an unprogrammed shift in balloon position, the illuminated area will be made to follow the image by changing the phase distribution over the array, thereby changing the angle of reception of the array.

A different adjustment is needed if the balloon attitude if disturbed. In this case, the disturbance moves the position at which the diffraction image is re-focussed by the secondary. This effect can be compensated by making the receiving array large enough to accommodate the change in position and by controlling the active area of the array to follow the change in position.

An array could be constructed with each antenna element connected to an amplifier, programmable phaseshifter and attenuator. A scheme for inter-nesting antenna elements, differing by a factors of two in operating wavelength, has been considered in the URSI sponsored study of a one square $\mathrm{km}$ array (Braun 1993). The adoption of this arrangement for the telescope proposed here would allow observations at several wavelengths simultaneously. Alternatively, mixers might be used to translate a number of different observing frequencies to a common intermediate frequency. A single set of phase shifters and other

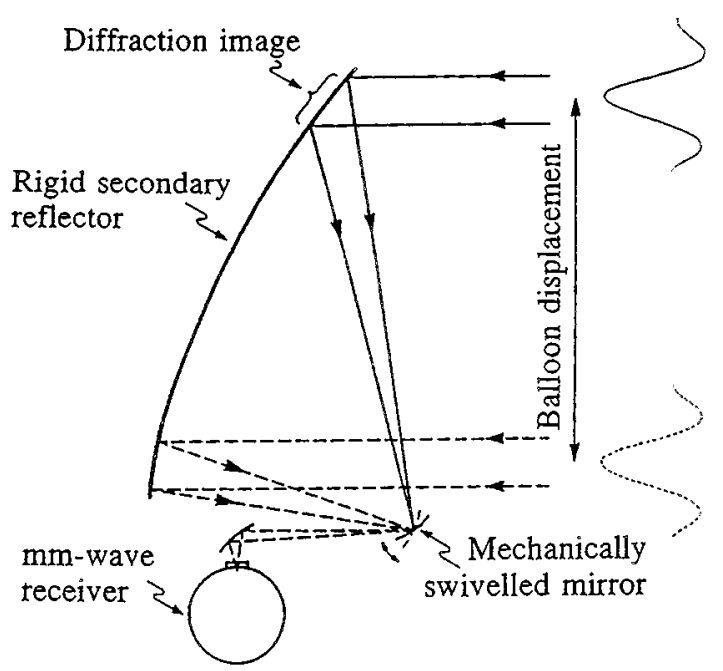

Fig. 6. A mechanically swivelled mirror used to correct for balloon motion at $\mathrm{mm}$ wavelengths

hardware would then allow, with minimum weight and cost, observations of several wavelengths one at a time. Division of the amplified signals amongst several sets of phase shifters might also be considered. This could give multiple beams at those wavelengths where the secondary is large compared to the diffraction image.

Low-noise amplifiers for a phased array are more difficult to obtain at $\mathrm{mm}$ wavelengths. Suitable amplifiers may soon be available, however, and at least one array for a radio telescope is being built for a $3 \mathrm{~mm}$ wavelength using HEMT amplifiers (Erickson 1995). In the meantime, the telescope feed for these short wavelengths could consist of a separate receiver combined with a mechanically swivelled mirror as sketched in Fig. 6. The mirror that is required is small, particularly for smaller diameter $\mathrm{mm}$ wavelength telescopes, and would be moved rapidly with a servo-mechanism to compensate for balloon motion. The optical arrangement requires that a fixed mirror near the receiver (Fig. 6) and the secondary reflector be located at, or near, conjugate foci of the swivelled mirror. Coma aberration, resulting from a mirror movement will be small because of the large $f / D$ of the mirror.

It is less clear how best to deal with changes in size and shape of a mm- wavelength diffraction image brought about by changing zenith angle. One possibility which may be practical is to mechanically change the ellipticity and size of a diverging (or converging) beam through the use of obliquely-angled cylindrical mirrors.

\subsection{Measurement of the position and attitude of the airborne vehicle}

A first step in controlling the airborne vehicle is to accurately measure its position and attitude. While there are probably many suitable schemes for doing this, a particular example is discussed here with the aim of showing that 
the required accuracy is attainable. The position accuracy needed is taken to be $1 / 20$ of the spacing between zeroes in the diffraction image of a point source for lateral position; $1 / 200$ of a wavelength for radial distance from the primary reflector (this stringency is needed to keep track of phase for aperture synthesis); and 1/20 of the angle subtended by the primary reflector at the receiver for balloon attitude relative to the focal axis. Balloon attitude around the focal axis is not critical and is assumed to be measured by an on-board inclinometer.

As a first stage in the measurement an on-board Global Positioning System (GPS) receiver, combined with a reference receiver on the ground, will provide a reliable position to an accuracy of about $3 \mathrm{~m}$. (Dooling 1994). As a second stage consider a system consisting of a $3 \mathrm{~m}$ diameter antenna located at the centre of the main reflector and equipped with the type of monopulse feed used for radar (see, for example, Jasik 1984). Two C.W. tones near $30 \mathrm{GHz}$ and differing in frequency by $10 \mathrm{MHz}$ are transmitted in the sum beam of the monopulse feed at a level of one watt. These signals are received at the airborne vehicle by an antenna that is isotropic over the lower $2 \pi$ sterradians. They are amplified, up-converted to $34 \mathrm{GHz}$ and re-transmitted to the ground at a level of $10^{-2}$ watts. The re-transmitted signals are rapidly switched between four antennas which are offset symmetrically from the focal axis: a pair separated by $1 \mathrm{~m}$ horizontally and a pair in the vertical plane of the source and also separated by $1 \mathrm{~m}$.

The GPS system is accurate enough to steer the balloon into the beam of the monopulse antenna (in the worst case, approximately $6 \mathrm{~m}$ to the half-power points at a distance of $1500 \mathrm{~m}$ ). The monopulse antenna is driven so that its axis points in the direction of the target position of the airborne vehicle. The lateral position of the vehicle is measured using horizontal and vertical differential outputs from the monopulse feed. Commands to correct the position of the vehicle are conveniently derived from these signals. With a bandwidth of $100 \mathrm{~Hz}$ and $300 \mathrm{~K}$ system noise temperature the monopulse receiver sensitivity is high. The noise level corresponds to a lateral displacement of less than $0.3 \mathrm{~cm}$ for the maximum distance of $R=6000 \mathrm{~m}$. This implies that the error would be determined not by noise but by how accurately the monopulse antenna can be pointed. Clearly, the same or better pointing is required for the monopulse antenna as is needed in pointing the complete radio telescope. At $\lambda=1.3 \mathrm{~cm}$, pointing to $1 / 10$ of the beamwidth of the $200 \mathrm{~m}$ telescope of Table 1 requires an accuracy of 1.5 arcsec.

The radial distance to the vehicle can be measured using the beat between the two $34 \mathrm{GHz}$ tones from the vehicle transponder. The phase of this $10 \mathrm{MHz}$ signal will give the radial distance with an ambiguity that can be resolved by the GPS position. A possibly important advantage of using two C.W. tones is that there need be no interfering sidebands or harmonics in the cm or decime-
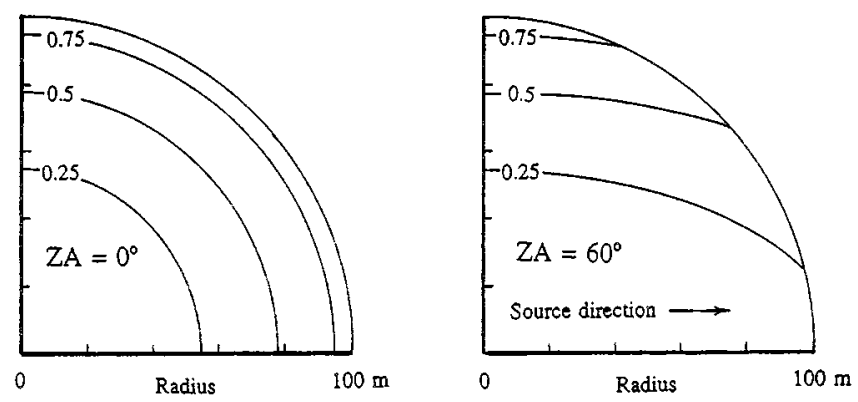

Fig. 7. Elevation contours required on the reflector surface of $200 \mathrm{~m}$ diameter are plotted in metres for a receiver altitude of $3000 \mathrm{~m}$ and zenith angles of $0^{\circ}$ and $60^{\circ}$

tre wavelength ranges or at $\mathrm{mm}$ wavelengths commonly used in astronomy. The calculated noise level for a $50 \mathrm{~Hz}$ bandwidth and $300 \mathrm{~K}$ system temperature corresponds to a change in radial distance of less than $0.005 \mathrm{~cm}$. We note that the measurement includes any change in atmospheric path-length from the primary reflector to the balloon.

Measuring radial distance to $0.005 \mathrm{~cm}$ for each of the four offset antennas on the balloon will determine the balloon attitude to about 10 arcsec. This is small compared to an accuracy of about 2.8 arcmin (1/20 of a subtended reflector diameter of $100 \mathrm{~m}$ at $6000 \mathrm{~m}$ ) that is actually needed to point the secondary reflector at the primary.

\subsection{The reflector surface}

The reflector surface will be made up of a large number of square panels as sketched in Fig. 1. The shape of the reflector that is needed depends on the angular position of the source. To describe this quantitatively, consider a radio source at azimuth angle $A Z$ and zenith angle $Z A$ and a rectangular coordinate system with the $z$-axis vertical and the $x$-axis horizontal and along the bearing of the source. The equation of the surface that will bring the source emission into a focus a distance $R$ from the centre of the surface and at zenith angle $Z A$ and azimuth $A Z+180^{\circ}$ is:

$$
\begin{aligned}
x^{2} \cos (Z A)+y^{2} \cos ^{-1}(Z A) & =4 R z(1+x(\sin (Z A) / 2 R)) \\
& \approx 4 R z \text { for } R \gg x
\end{aligned}
$$

As a special case, it is useful to consider tracking a radio source while keeping the airborne vehicle at a constant altitude. This minimizes the adjustment of the surface and the actuator travel needed. If, for example, the vehicle is kept at a constant altitude: $h_{0}=R \cos (Z A)$, the equation of the required reflector surface becomes:

$$
\begin{aligned}
(x \cos (Z A))^{2}+y^{2} & =4 h_{0} z\left(1+x\left(\sin (Z A) \cos (Z A) / 2 h_{0}\right)\right) \\
& \approx 4 h_{0} z \text { for } h_{0} \gg x
\end{aligned}
$$

This case of constant receiver altitude is illustrated in Fig. 7 where contours of surface elevation are drawn for a 


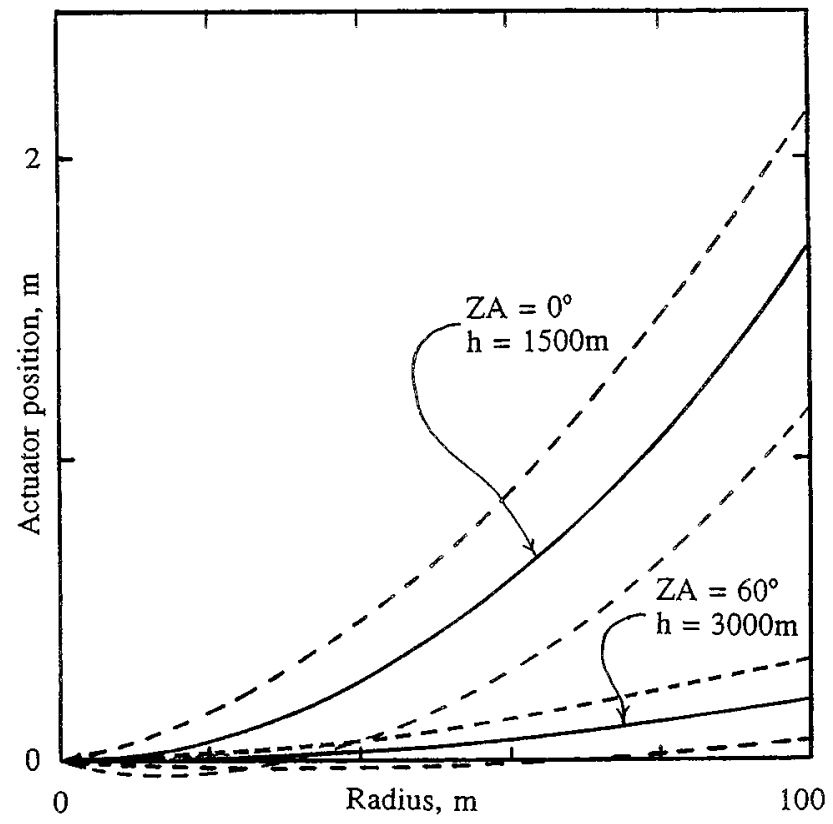

Fig. 8. The surface profiles, or the required actuator positions, for a $200 \mathrm{~m}$ diameter reflector along the azimuth of the source. Solid lines show the profiles for the balloon at the ideal target position. Dashed lines show the adjustment in the profiles needed to move the diffraction image $\pm 15 \mathrm{~m}$ at the balloon

reflector of $200 \mathrm{~m}$ diameter, an altitude of $h_{0}=3000 \mathrm{~m}$ and for source zenith angles of $0^{\circ}$ and $60^{\circ}$. (The asymmetry of $z$ with $x$ amounts to less than one centimetre in $z$ and is ignored in Fig. 7). The largest changes in elevation of the surface are required on the outside panels along an axis at the (changing) bearing of the radio source. Some of the outside panels need to be lowered from an elevation of $z=0.83 \mathrm{~m}$ to an elevation of $z=0.21 \mathrm{~m}$ as the source zenith angle changes from $0^{\circ}$ to $60^{\circ}$. For the same change in zenith angle but a receiver altitude of $1500 \mathrm{~m}$, the outside panels need to be moved twice as far, from $1.67 \mathrm{~m}$ to $0.42 \mathrm{~m}$.

The panels also need to be moved to compensate for possible erratic motion of the balloon. In general, to move the diffraction image through $\pm W / 2$ metres at a distance $R$ requires an actuator adjustment of:

$A= \pm W D / 8 R=( \pm W D \cos (Z A)) / 8 h$.

Examples of the primary reflector profile that is required are drawn in Fig. 8, showing the actuator adjustment needed to accommodate a change of zenith angle, a change of altitude and a change in the balloon position of $\pm 15 \mathrm{~m}$. Extremes in the profile are found at minimum altitude and zero zenith angle compared to maximum altitude and maximum zenith angle. The difference between these extremes is the actuator adjustment expressed by Eq. (1). We note that actuators closer to the centre of the reflector require less travel and may as a result be less expensive.
Regardless of telescope design, if a telescope is large enough (and the site bad enough) there is the possibility of atmospheric phase fluctuation over its aperture. It is interesting to note that, if the phase fluctuation is measurable in real time (holographically, for example, if the telescope is part of a synthesis array), it can in principal be corrected with an actuator-controlled surface of the type described here.

The use of individual square panels (Fig. 1) appears to provide a straightforward and inexpensive way of constructing a large reflecting surface. There are however other possibilities that might be still less expensive. One type of surface that has been considered is made up of very long (e.g $100 \mathrm{~m}$ or more), flexible reflecting strips that are several metres wide and suspended side by side. The strips are supported at their ends by actuators or other devices of adjustable height. One actuator per strip sets the tension in a strip and thereby the focal length. Such an arrangement can, at least in principle, form an adjustable reflecting surface of the type required. With practical values of tension in the strips the deviation of the catenary profile from a parabola is a fraction of a cm and can, with some added complication, be corrected for very short wavelengths. The number of actuators needed for this surface is much smaller than the number needed to support individual panels, increasing only in proportion to the reflector perimeter, rather than to area. A disadvantage of the surface is that some form of protection might be needed against wind.

\subsection{Individual reflector panels}

The reflector panels need to be as large as possible to minimize both the number of panels and the number of actuators required. The maximum size is determined by path-length errors and can be increased by giving the panels a slight curvature. From Eq. (9), the surface profile required at constant receiver altitude $h_{0}$ is:

$z=y^{2} / 4 h_{0}$

in the $y-z$ plane and:

$z=y^{2} \cos ^{2}(Z A) / 4 h_{0}$

in the $x-z$ plane. Ideal surface panels would have radii of curvature of $\rho_{1}=2 h_{0}$ in the $y-z$ plane and $\rho_{1}=$ $2 h_{0} \sec ^{2}(Z A)$ in the $x-z$ plane. A reasonable compromise for $0^{\circ} \leq Z A \leq 60^{\circ}$ is a curvature giving the same pathlength errors at $Z A=0^{\circ}$ and $Z A=60^{\circ}$. For $h_{0}=3000$ $\mathrm{m}$ the required radius of curvature is found to be $9600 \mathrm{~m}$.

The effect of path-length errors is found using results calculated for a flat, square panel which are shown in Fig. 9. For a panel with a radius of curvature $\rho$, the effective receiver distance, which can be used with Fig. 9, is increased from an actual distance, $R$, to an effective distance:

$R_{1}=0.5 \rho \rho_{1} /\left|\rho-\rho_{1}\right|$, 


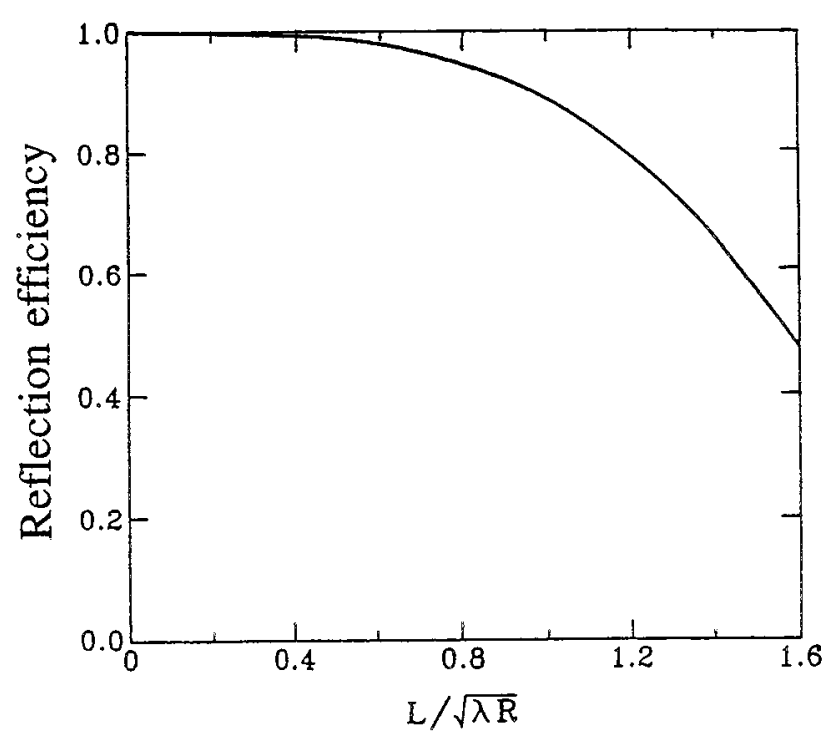

Fig. 9. The calculated reflection efficiency for a flat, square reflector of side dimension $L$, with wavelength and reflector to point-of-measurement distance $R$. A plane wave is assumed incident. The curve shows the decrease in power density caused by path-length differences for the flat reflector compared to a perfectly focussed reflector

where $\rho_{1}$ is the ideal radius of curvature. It is found that for $\lambda=2.8 \mathrm{~cm}$, a panel of side dimension $L=10 \mathrm{~m}$ and the $9600 \mathrm{~m}$ radius of curvature give a reflection efficiency of more that 0.97 over the whole zenith angle range. (Equation (5) of Sect. 3.1 is approximately satisfied for $\lambda=2.8 \mathrm{~cm}$ with the actual receiver distance $3000 \mathrm{~m}$ ). For $\lambda=1.3 \mathrm{~cm}$, the $10 \mathrm{~m}$ panel gives a reflection efficiency greater than 0.89 for zenith angles up to 60 degrees.

The reflecting surface of the panels might be formed from a thin continuous or perforated aluminum sheet. For a $10 \mathrm{~m}$ panel, it is difficult to put an unsupported sheet under enough tension to achieve a radius of curvature as large as $9600 \mathrm{~m}$. Heavy compression members would be needed in each panel to sustain the tension. The screen will for this reason have to be supported in several places across a $10 \mathrm{~m}$ span. For good efficiency at $\lambda=1.3 \mathrm{~cm}$ there will also need to be elevation adjustments at the points of support to bring the reflector to within about $0.8 \mathrm{~mm}$ of the ideal surface. Deep, rigid trusses are needed and these will have to be made light to keep the cost low. An initial study suggests that the design might use cables as efficient tension members on the underside of the trusses, possibly similar to the sketch in Fig. 1.

Smaller panels are required at mm-wavelengths. In this case, because of the smaller size, it is possible that panels can be made by stretching thin sheets of metal between two opposite sides as drawn in Fig. 10. The reflecting surface is then defined by two narrow edges of a supporting frame. The edges need to be accurately machined but they can be straight and their area can be less than $1 \%$ of the panel area. Other parts of the panel do not need to be

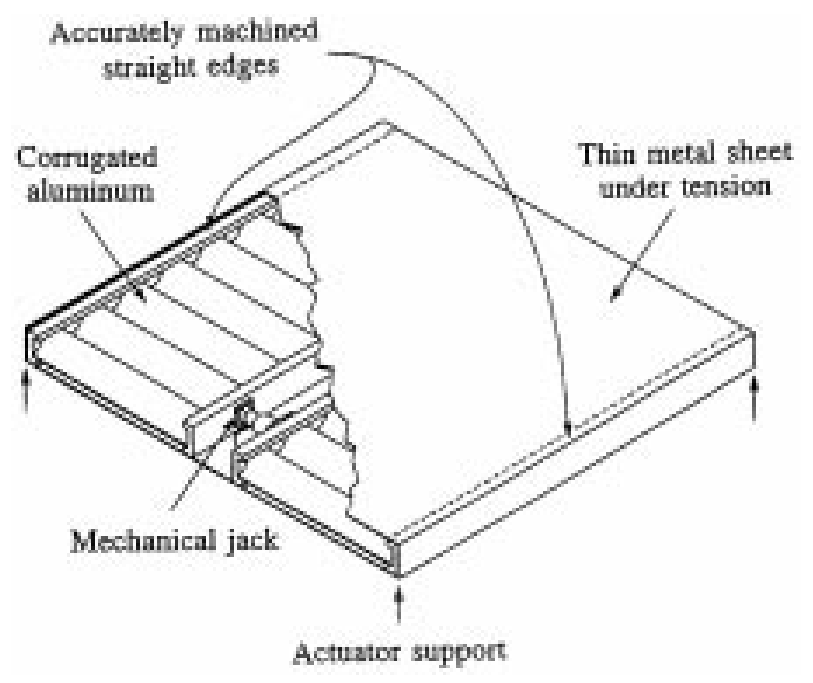

Fig. 10. A possibly inexpensive means of constructing a panel for $\mathrm{mm}$ wavelengths

accurately constructed and the machined edges can be made to lie in a plane by a screw adjustment at the panel corners. This form of construction may allow accurate surfaces to be made with little precision machining and is a consequence of the long focal length of the telescope.

If the mm-wave reflecting sheets are highly stressed their sag under gravity will be small and their vertical profile will be almost exactly a circular arc. A calculation shows that a square panel $1.6 \mathrm{~m}$ on a side, with a radius of curvature of $2000 \mathrm{~m}$, gives a reflection efficiency of about 0.95 at a wavelength of $1.3 \mathrm{~mm}$ for a feed at a distance of $3000 \mathrm{~m}$. The efficiency is the same if the panel is perfectly flat $(R=\infty)$ and better than 0.95 for $2000 \leq R \leq \infty$. The stress required in an aluminum sheet to give a radius of curvature of $2000 \mathrm{~m}$ or more is an acceptable $5.3 \times$ $10^{8}$ dynes $\mathrm{cm}^{-2}$, about one quarter the tensile strength of aluminum alloy.

\section{Conclusions}

Several conclusions can be drawn from the present study. First, there should be large cost savings in constructing a primary surface supported by the ground compared with constructing a conventional steerable paraboloidal surface. Motion of the surface panels is almost entirely a vertical displacement, involving no change in gravitational loading. Thermal effects should be well balanced over the surface, and should be small because of the small vertical dimensions of the structure. The surface should therefore be stable. As a result, panels can be large but lightly constructed. Adjustment to improve their shape will be independent of zenith angle and should give a permanent benefit.

A further result of the stability, and a consequence of having each panel supported by the ground, is that no radome would be needed for $\mathrm{mm}$ wavelengths. Also, it 
may be possible to make accurate mm-wavelength panels with inexpensive materials and little precision machining as sketched in Fig. 10. The feasibility of this type of construction can be determined by building one panel for testing. A disadvantage of the telescope at $\mathrm{mm}$ wavelengths is the greater atmospheric loss that results from the very long focal length. However, the loss is very small compared to the potential gain in collecting area. Even a single telescope array element $110 \mathrm{~m}$ in diameter (Table 3) would have over 10 times the collecting area of the IRAM $30 \mathrm{~m}$ telescope, the largest existing $\mathrm{mm}$-wave instrument. This compares with an estimated $4 \%$ loss for one complete atmosphere (a 10 to $12 \mathrm{~K}$ atmospheric temperature contribution) from microwave background measurements at $\lambda=3.3 \mathrm{~mm}$ (Smoot et al. 1985).

The principle risk associated with the telescope is clearly in the positional stability of the balloon. It is believed that the stability problem may be solved by having (i) smaller scale balloon motion rapidly corrected by moving the feed point electronically and (ii) larger scale motion corrected (less often) by moving the diffraction image to follow the balloon (iii) six-axis control of the balloon position and attitude and (iv) a good site without mountains or unevenly heated ground. A computer simulation of balloon motion using existing meteorological data on winds would go a long way in demonstrating the effectiveness of these measures. A second step would be to use the simulation result in actually constructing a balloon with a six-axis control system and testing it at a good site

Acknowledgements. I thank the referee for his comments, particularly in pointing out the change in efficiency that can result from having the diffraction image follow the balloon and the importance of illumination spillover.

\section{References}

Beard J., 1994, New Scientist 144 (number 1947), 26

Blum E.J., Boischot A., Lequeux J., 1963, Proc. IRE Australia, 24, 208

Braun R., 1993, A Next Generation Radio Observatory: The Square Km Array, URSI General Assembly, Kyoto, Japan

Braun R., 1996, in: Jackson N. \& Davis R. (eds.) High Sensitivity Radio Astronomy. Cambridge Univ. Press

Clark A., 1988, Canadair Limited, Montreal, Quebec, Canada (private communication)

Colting H., 1994, 21st Century Airships Inc., Newmarket, Ontario, Canada (private communication)

Dooling D., 1994, IEEE Spectrum 31, 24

Erickson N.R., 1995, in: Emerson D.T. \& Payne J.M. (eds.) Multi-Feed Systems For Radio Telescopes, PASPC, 207

Gabuzda D.C., Cawthorne T.V., Roberts D.H., Wardle J.F.C., 1992, ApJ 388, 40

Gordon W.E., Lalonde L.M., 1961, IRE Trans. Antennas Propag. AP-9, 17

Jasik H.J., 1984, Antenna Engineering Handbook, chapter 34. McGraw Hill

Kemball A.J., Diamond P.J., Pauliny-Toth I.I.K., 1996, ApJ 464, L55

Korolkov D.V., Pariiskii Yu.N., 1979, Sky and Telescope 57, 324

Kraus J.D., Nash R.T., Ko H.C., 1961, IRE Trans. Antennas Propag. AP-9, 4

Legg T.H., 1996, in: Jackson N. \& Davis R. (eds.) High Sensitivity Radio Astronomy. Cambridge Univ. Press

Reber G., 1940, Proc. IRE 28, 68

Reid R.D., Moran, J.M., 1988, in: Verschuur G.L. \& Kellermann K.I. (eds.) Galactic and Extragalactic Radio Astronomy, 2nd Ed. Springer-Verlag, p. 292

Silver S., 1949, Microwave Antenna Theory and Design. McGraw Hill

Smoot G.F., et al., 1985, ApJ 291, L23 